\title{
Impact of proton implantation parameters on the photoconductivity of photorefractive multiple quantum wells
}

\author{
Błażej Jabłoński ${ }^{* 1}$ Agnieszka Branecka ${ }^{1}$, Eliza Miśkiewicz ${ }^{1}$, Andrzej Ziółkowski ${ }^{1}$, Ewa Weinert-Rączka ${ }^{1}$ \\ ${ }^{1}$ West Pomeranian University of Technology in Szczecin, Piastów 17, 70-310 Szczecin,
}

Received November 26, 2015; accepted December 22, 2015; published December 31, 2015

\begin{abstract}
Semi-insulating GaAs/AlGaAs multiple quantum wells are photorefractive materials with high sensitivity and short response time. Semi-insulation of these structures is commonly obtained by proton implantation. We present the measurements of photoconductivity in the samples with different proton doses. The results were compared to the theoretically predicted relationship between photoconductivity and the donor to acceptor concentration ratio. This allows to estimate the impact of proton dose on deep donor concentration.
\end{abstract}

The photorefractive effect is an optically induced change in the refractive index due to non-homogeneous illumination [1-2]. The material in which the photorefractive effect can be obtained should exhibit sufficiently high dark resistivity, photoconductivity and electro-optic effect. In the case of photorefractive semiconductors resistivity is usually increased by proton irradiation, which creates defects in the structures. It is essential to estimate the influence of proton implantation on various properties of the structure. This study presents the current-voltage characteristics of uniformly illuminated photorefractive multiple quantum wells (PRMQW) that were treated by different doses of protons. The experimental results have been compared to a theoretically predicted dependence of current density on electric field for various defect concentrations.

The commonly used model of a photorefractive phenomenon in quantum wells assumes that two types of 'defect' levels occur in the energy gap: deep donors with concentration $N_{D}$ and shallow acceptors with concentration $N_{A}$. The presence of shallow levels is the reason why the structure before implantation had relatively strong dark conductivity. Proton implantation creates deep levels that compensate for shallow dopants existing in the material and act as traps in photorefractive effect.

When an electric field with intensity above the critical value $E_{A} \approx 3 \mathrm{kV} / \mathrm{cm}$ is applied to a PR-MQW structure, nonlinear phenomena due to changes in electron mobility begin to take place. These phenomena turn out to be crucial from both cognitive and practical perspectives, affecting photorefractive gain during wave mixing [3-5] and non-linear propagation of light beams in

*E-mail: blazej.jablonski@zut.edu.pl semiconductors [6]. Changes in the mobility result from increasing energy of electrons, which is described as the increase of electron temperature that gets much higher than the temperature of lattice $T_{e} \gg T_{L}$. Electron temperature can be expressed by the relation [7]:

$$
T_{e}(E)=T_{L}+\frac{2 \mathrm{q} \tau_{r} \mu_{e}(E)}{3 \mathrm{k}_{\mathrm{B}}} E
$$

where $\mu_{e}(E)$ is the mobility of electrons dependent on electric field intensity, $\tau_{r}$ is the mean energy relaxation time, $\mathrm{q}$ is the elementary electric charge, and $\mathrm{k}_{\mathrm{B}}$ is the Boltzmann constant.

In the conductivity band of semiconductors such as GaAs, apart from the central valley there are side valleys characterized by lower mobility. When the intensity of an electric field applied to the structure exceeds the value $E_{A}$, a part of the electrons is transferred from the central valley to side valleys. This is usually described by a 'two valleys' model, in which electron mobility is expressed as the weighted average:

$$
\mu(E)=\mu_{e l} f(E)+\mu_{e u}[1-f(E)]
$$

where $\mu_{e l}$ and $\mu_{e u}$ are electron mobilities in, respectively, central valley and side valley, while $f(E)$ is a distribution function determining the occupancy of the central valley [7].

$$
f(E)=\left\{1+R \cdot \exp \left[\frac{-\Delta U}{\mathrm{k}_{\mathrm{B}} T_{e}(E)}\right]\right\}^{-1}
$$

where $\Delta U$ describes the difference in energy between the central and satellite minima, $R$ is the ratio of densities of states in central and side valleys; (for GaAs: $R=94$, $\Delta U=0.31 \mathrm{eV})$.

The tested structures, made at the Institute of Electronic Materials Technology in Warsaw, were built of thin alternately laid GaAs layers 7-nm-thick, acting as wells, and $\mathrm{Al}_{0.3} \mathrm{Ga}_{0.7} \mathrm{As}$ layers 6 -nm-thick acting as barriers. The total thickness of the PR-MQW thin film was $1.3 \mu \mathrm{m}$. They were subjected to proton implantation of different doses. Each sample underwent implantation twice, each time with different protons energies, as shown in Table 1. 
Table 1. Values of energy and doses used during proton implantation in a PR-MQW structure.

\begin{tabular}{|c|c|c|c|c|}
\hline Structure & Energy 1 & Dose 1 & Energy 2 & Dose 2 \\
\hline 6A & \multicolumn{4}{|c|}{ WITHOUT IMPLANTATION } \\
\hline 6B & $160 \mathrm{keV}$ & $1 \times 10^{12} \mathrm{~cm}^{-2}$ & $80 \mathrm{keV}$ & $1 \times 10^{12} \mathrm{~cm}^{-2}$ \\
\hline $\mathbf{6 C}$ & $160 \mathrm{keV}$ & $1.5 \times 10^{12} \mathrm{~cm}^{-2}$ & $80 \mathrm{keV}$ & $1.5 \times 10^{12} \mathrm{~cm}^{-2}$ \\
\hline 6D & $160 \mathrm{keV}$ & $2 \times 10^{12} \mathrm{~cm}^{-2}$ & $80 \mathrm{keV}$ & $2 \times 10^{12} \mathrm{~cm}^{-2}$ \\
\hline
\end{tabular}

The laboratory setup was composed of an argonkrypton laser which generated wavelength $\lambda=647 \mathrm{~nm}$, beam intensity control system, measurements of currentvoltage characteristics setup and a PR-MQW structure. An electric field was applied parallel to the planes of quantum wells (Franz-Keldysh geometry).

Figure 1 depicts the current-voltage characteristics of the tested structures without illumination. One can see that structure 6A, not subjected to implantation, behaves like a semiconductor, while in the structures $6 \mathrm{~B}-6 \mathrm{D}$ the current intensity is smaller and has quasi-linear characteristic.

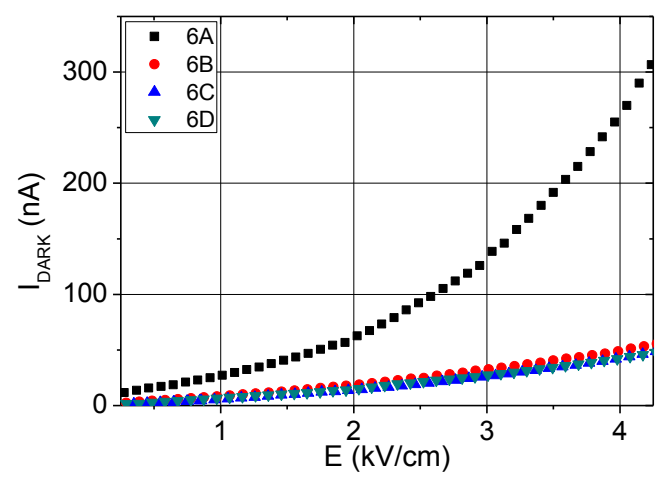

Fig. 1. Dark current as a function of the applied field for the structures, with different proton doses.

Typical current-voltage characteristics of structures illuminated with a homogeneous light beam are presented in Fig. 2.
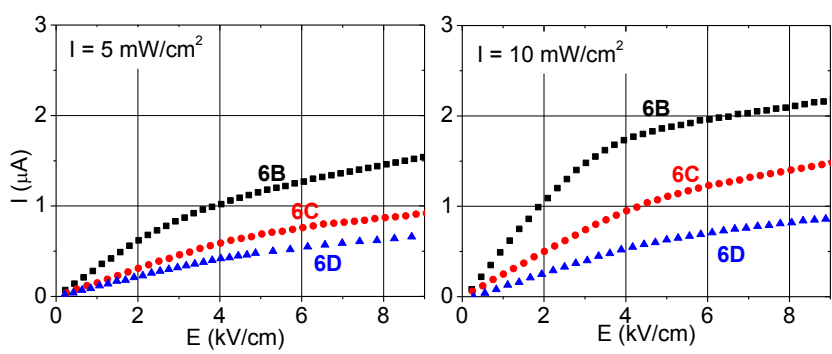

Fig. 2. Photocurrent as a function of the applied field for the structures $\mathrm{B}, \mathrm{C}$ and D. Light intensity (a) $\mathrm{I}=5 \mathrm{~mW} / \mathrm{cm}^{2}$ (b) $\mathrm{I}=10 \mathrm{~mW} / \mathrm{cm}^{2}$.

The impact of proton implantation on current-voltage characteristics is visible. The higher the proton dose is, the lower the value of current intensity we get. Assuming that the generation of deep defects is the main effect of implantation, the observed decrease of dark resistivity can be explained by better compensation of shallow defects present in the material while decrease of photocurrent by increasing density of traps for photo-generated carriers. For electric field intensity above critical value $E_{A}$, the characteristic curvature related to nonlinear transport of electrons is seen.

The total density of current in photorefractive multiple quantum wells can be written as the sum of electron and hole current densities:

$$
j=j_{e}+j_{h}=\mathrm{q}\left[\mu_{e}(E) n_{e}+\mu_{h} n_{h}\right] E_{0}
$$

where $\mu_{\mathrm{e}}$ and $\mu_{\mathrm{h}}$ represent the mobility of electrons and holes, $n_{e}$ and $n_{h}$ denote the concentration of electrons and holes for homogeneous illumination [3-4, 9]. Carries concentrations in uniformly illuminated structures are:

where

$$
\begin{gathered}
n_{e}=(\alpha / \mathrm{hv}) I_{0} \tau_{e}, \\
n_{h}=(\alpha / \mathrm{hv}) I_{0} \tau_{h},
\end{gathered}
$$

$\tau_{e}=1 / \gamma_{e} N_{D}^{+}, \tau_{h}=1 / \gamma_{h} N_{D}^{0}$

are average recombination times for electrons and holes.

Table 2. Material parameters used in calculation.

\begin{tabular}{ll}
\hline$\alpha=5 \cdot 10^{3} \mathrm{~cm}^{-1}$ & interband absorption coefficient \\
$\gamma_{e}=4 \cdot 10^{-7}$ & trapping coefficient for electrons \\
$\gamma_{h}=2 \cdot 10^{-7}$ & trapping coefficient for holes \\
$\tau_{r}=10^{-12} \mathrm{~s}$ & energy relaxation time \\
$\mu_{e l}=5000 \mathrm{~cm}^{2} / V S$ & electron mobility in central valley \\
$\mu_{e u}=400 \mathrm{~cm}^{2} / V S$ & electron mobility in side valleys \\
$\mu_{h}=400 \mathrm{~cm}^{2} / \mathrm{Vs}$ & hole mobility \\
$\mathrm{I}_{0}$ & light density \\
$N_{A}$ & concentration of acceptors \\
$N_{D}$ & concentration of donors \\
$N_{D}^{+} \approx N_{A}$ & concentration of ionized donors which are trap \\
$N_{D}^{0}=N_{D}-N_{D}^{+}$ & center for electrons \\
& concentration of non-ionized donors which are \\
\hline
\end{tabular}

The lifetime of free carriers in photorefractive structures is short, $\tau_{e}, \tau_{h}<100 n s$ [10]. Owing to this we can assume that excited carriers are almost instantly trapped, which results in $N_{D}^{+} \approx N_{A}$. The donor compensation coefficient $r=N_{A} / N_{D}$, allows estimating whether the transport of carriers is dominated by electrons or by holes. When $r<0.34$, the transport is dominated by electrons, while for values $r>0.34$ by holes, which is shown in Table 3.

Table 3. Ratio of hole to electron concentrations in reference to the compensation coefficient.

\begin{tabular}{|c|c|c|c|c|c|c|c|c|c|}
\hline$r$ & 0.01 & 0.1 & 0.3 & 0.333 & 0.4 & 0.5 & 0.7 & 0.9 & 0.99 \\
\hline$\frac{n_{h}}{n_{e}}$ & 0.02 & 0.22 & 0.85 & 0.999 & 1.33 & 2 & 4.67 & 18 & 198 \\
\hline
\end{tabular}


Figure 7 presents theoretical characteristics of current intensity as a function of the electric field for different donor compensation coefficients. These calculations were made for homogeneous illumination $\mathrm{I}=10 \mathrm{~mW} / \mathrm{cm}^{2}$ and the standard value of acceptor concentration $N_{A}=3 \cdot 10^{16} \mathrm{~cm}^{-3}[2]$.

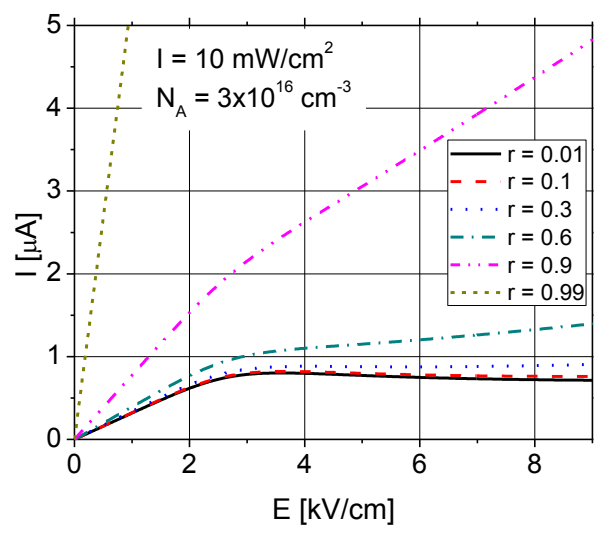

Fig. 7. Photocurrent as a function of the applied field, calculated for the acceptor concentration $N_{A}=3 \cdot 10^{16} \mathrm{~cm}^{-3}$ and different values of $\mathrm{r}$. Light intensity was $\mathrm{I}=10 \mathrm{~mW} / \mathrm{cm}^{2}$ and wavelength $\lambda=647 \mathrm{~nm}$.

Changes in shape of the curves are visible. As the donor compensation coefficient decreases, the curvatures of the characteristics for $E>E_{A}$ get larger, which is caused by increasing influence of nonlinearity of electron transport. Figure 8 shows photocurrent-voltage characteristics averaged for samples with the same doses of proton implantation. The solid lines represent analytical solutions, while the dots represent the experimental results, each determined from measurements of few samples.

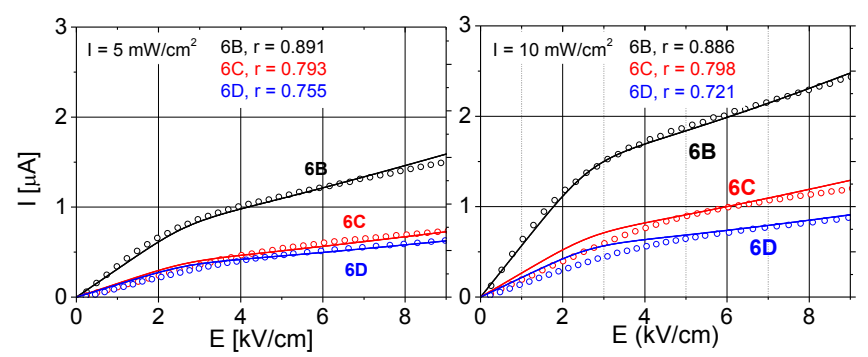

Fig. 8. Photocurrent as a function of the applied field for $N_{A} \approx 4.5 \cdot 10^{16} \mathrm{~cm}^{-3}$, different donor compensation ratio $r=N_{D} / N_{A}$ and two values of light intensity: (a) $\mathrm{I}=5 \mathrm{~mW} / \mathrm{cm}^{2}$, (b) $\mathrm{I}=$ $10 \mathrm{~mW} / \mathrm{cm}^{2}$. The circles denote measured values, and the plotted lines are analytical solutions.

The comparison of experimental and theoretical curves show that the donor compensation coefficient $r$ depends on proton dose, and for the applied implantation parameters ranges from 0.7 to 0.9 , which corresponds to the ratio of hole to electron concentrations $n_{h} / n_{e} \approx 5 \div$ 16. This indicates the dominance of hole transport in all tested samples.
In summary, the experiments and the theoretical analysis have been aimed at examining the impact of proton implantation in PR-MQW structures on photocurrent-voltage characteristics. By comparing experimental results to the analytical solution describing the intensity of current in the structure, we have estimated an approximate value of the donor compensation coefficient and the type of dominant carriers. All tested structures showed the dominance of holes over electrons. Nevertheless, we have observed the occurrence of nonlinear transport leading to a curvature of the characteristics for electric field intensity above the critical value $\left(E_{A}=3 \mathrm{kV} / \mathrm{cm}\right)$. It has been shown that by varying the doses of protons implanted in the structure we can, to some extent, affect the ratio of electron to hole concentrations. However, this ratio can be adjusted to specific needs only in a limited scope.

\section{References}

[1] D.D. Nolte, J. Appl. Phys. 85, 6259 (1999).

[2] D.D. Nolte, M.R. Melloch, in Photorefractive effects and Materials, ed. D.D. Nolte (Kluwer, Dordrecht, 1995).

[3] Q. Wang, R.M. Brubaker, D.D. Nolte, M.R. Meloch, J. Opt. Soc. Am. B 9, 1626 (1992).

[4] Q. Wang, RM. Brubaker, D.D. Nolte, J. Opt. Soc. Am. B 11, 1773 (1994).

[5] M. Wichtowski, A. Ziolkowski, E. Weinert-Rączka, J. Opt. 12, 065201 (2010).

[6] A. Ziółkowski, Opt. Expr. 22, 4599 (2014).

[7] K. Seeger, Semiconductors Physics, fifth ed., Chapter 1 (SpringerVerlag, Berlin, 1991).

[8] S.M. Sze, Physics of Semiconductors Devices, Second ed., Chapter 1 (Wiley, New York, 1981).

[9] S. Balasubramanian, I. Lahiri, Y. Ding, M.R. Melloch, D.D. Nolte, Appl. Phys. B 68, 863 (1999).

[10] P. Yeh in Introduction to Photorefractive Nonlinear Optics, (Wiley, New York, 1993). 